\title{
II CONGRESO SOBRE ARCHIVOS ECONOMICOS DE ENTIDADES PRIVADAS
}

TERESA TORTELLA CASARES

Archivo del Banco de España

Cuatro años después de la celebración del I Congreso sobre Archivos Económicos de Entidades Privadas, el Archivo del Banco de España ha convocado un II Congreso sobre este mismo tema. En aquella ocasión (3-4 junio 1982) el Congreso se organizó en el marco de la conmemoración del bicentenario de la fundación del Banco Nacional de San Carlos. Como ponentes figuraron destacados archiveros e historiadores, entre los que se encontraba don Ramón Carande. Tuvo, entonces, una gran acogida de público, que puso de manifiesto el interés que existe en nuestro país no ya por la Historia Económica, sino también por un mayor conocimiento de nuestros archivos.

Este II Congreso, celebrado en la Sala de Investigadores del Archivo del Banco de España, durante los días 26 y 27 de junio de 1986, se ha presentado ya con un título específico: «Fuentes para la historia de la banca y el comercio en España». Nuevamente los participantes pertenecen, en su mayoría, al mundo de los archivos y de la Universidad. Esta vez, además de contar con una representación importante de colaboradores españoles, figuraban también cuatro profesores venidos de más allá de nuestras fronteras, todos ellos vinculados de forma muy directa con los archivos: Albert Broder, Rondo Cameron, Hans Pohl y David Ringrose.

Como continuación del anterior, este II Congreso tenía como finalidad, una vez más, facilitar el intercambio de conocimientos entre archiveros e historiadores acerca de estos temas. Pero, al mismo tiempo, se proponía impulsar nuevos estudios sobre la banca española y estimular a las entidades propietarias de los archivos para que permitan el acceso a éstos y se abran así nuevas vías de investigación. El Congreso se desarrolló en cuatro sesiones de trabajo. También en esta ocasión tuvo una gran acogida de público, que nuevamente volvió a llenar la Sala de Investigadores.

El tema general de la primera sesión de trabajo fue «Comerciantes y banqueros en el Antiguo Régimen». Como moderador actuó Felipe Ruiz Martín, catedrático de Historia Económica de la Universidad Autónoma de Madrid. 
Los ponentes fueron Esteban Hernández Esteve, Margarita Cuartas Rivero, David Ringrose y Pedro Tedde de Lorca.

Esteban Hernández, director del Centro de Formación del Banco de España, en sus "Aportaciones al estudio de la banca castellana en el siglo xvI", habló sobre los proyectos de creación de bancos municipales en las principales ciudades castellanas, así como de uno concreto de Sevilla. Estos proyectos no llegaron a realizarse, pero existe una amplia información sobre ellos en el Archivo de Valencia de Don Juan y en el de Zabálburu o de Heredia Spínola.

Margarita Cuartas, directora del Archivo Histórico Provincial de Gerona, presentó una ponencia sobre «Los banqueros y el medio general de 1608: organización y documentación en la Sección Dirección General del Tesoro del Archivo de Simancas». Su contenido recoge detalladamente la organización y operaciones de los hombres de negocios con los que la Real Hacienda estableció un concierto económico para satisfacer la deuda de la Corona tras la suspensión de pagos de diciembre de 1607.

David Ringrose, catedrático de Historia de la Universidad de California, San Diego, expuso a continuación los resultados de su investigación que tiene por título "Hombres de negocios y hombres de la política en el Madrid de la primera mitad del siglo xIx». Su trabajo está dedicado a estudiar las características principales de la sociedad madrileña en este tiempo, y en especial de dos clases social y económicamente sobresalientes: la de los comerciantes y la de los políticos.

Pedro Tedde, historiador del Servicio de Estudios del Banco de España y catedrático de Historia Económica de la Universidad de Málaga, cerró la sesión con su ponencia sobre «Las fuentes documentales para el estudio del origen de la banca en el siglo xvirr: fondos del Banco de San Carlos». Se refirió a los archivos que conservan documentación sobre el primer banco nacional español, describiendo los fondos más valiosos para el estudio de cada uno de los seis grandes períodos en que él divide los cincuenta años de la historia de este banco. Además del Archivo del Banco de España, aludió al Archivo Histórico Nacional y al de Simancas.

La segunda sesión, que tuvo lugar el día 26 por la tarde, estuvo dedicada a «Archivos bancarios de diferentes ámbitos geográficos». El moderador de la misma fue Nicolás Sánchez Albornoz y las ponencias fueron presentadas por Antonio Mut Calafell e Isabel Garau Llompart; Carles Sudriá; Francesc Cabana; Rafael Anes; Xan Carmona, Olga Gallego y Pedro López, y, por último, Rafael Castejón y Manuel Titos.

Antonio Mut Calafell, director del Archivo del Reino de Mallorca, e Isabel Garau Llompart iniciaron la sesión con un trabajo sobre los «Fondos privados de carácter económico del Archivo del Reino de Mallorca y de otros 
archivos mallorquines». Antonio Mut describió los diversos fondos documentales de carácter familiar y privado conservados en el Archivo del Reino de Mallorca. Isabel Garau, a su vez, habló de un conjunto de sociedades de ahorro mallorquinas de la segunda mitad del siglo xix y principios del $\mathbf{x x}$.

Las dos ponencias siguientes estuvieron dedicadas a la historia de la banca catalana y las fuentes para su estudio. En primer lugar, Carles Sudriá, profesor titular de Historia Económica de la Universidad de Barcelona, expuso el tema de las «Fuentes para el estudio de la actividad de las entidades bancarias catalanas en el siglo xix». Destacó la importancia de los archivos del Estado, de los archivos judiciales y de los de corporaciones de empresarios como fuentes alternativas valiosas para el estudio de la banca, teniendo en cuenta la falta de testimonio acerca de bancos y banqueros desaparecidos.

En cuanto a Francesc Cabana, cuyo trabajo tiene por título «Bases de investigación y archivos para la historia bancaria catalana en el siglo XX», deslindó cuatro grandes campos de investigación bancaria en Cataluña y se refirió a las dificultades que se le presentan al investigador de hoy para conocer la documentación de los archivos de las sociedades actuales con raíces en el pasado, así como de aquellas entidades que han sido absorbidas por otras.

Rafael Anes, catedrático de Historia Económica de la Universidad de Oviedo, habló sobre «Casas de banca asturianas y sus fondos documentales». En el mismo sentido que Carles Sudriá para Cataluña, indicó la importancia que tienen las matrículas de comerciantes y registros mercantiles para suplir la falta de documentación de casas de banca y banqueros asturianos desaparecidos. Ofreció, además, una relación detallada de sociedades bancarias que desarrollaron sus actividades en Asturias en los siglos xIX y XX.

Xan Carmona, profesor titular de Historia Económica de la Universidad de Santiago; Olga Gallego, directora del Archivo Histórico-Provincial de Orense, y Pedro López Gómez, director del Archivo del Reino de Galicia, son los autores de la ponencia titulada «Fuentes para la historia de la banca en Galicia durante el siglo xIX y principios del XX", que fue presentada a continuación por Xan Carmona. Se refirió a los principales bancos gallegos de los que se sabe que conservan papeles y, al mismo tiempo, aludió a las principales fuentes indirectas, que, en muchos casos, son las únicas disponibles, dada la ausencia de fuentes básicas que se registra en Galicia.

Manuel Titos Martínez, profesor titular de Historia Contemporánea de la Universidad de Granada y subdirector general de la Caja de Ahorros de esta misma ciudad, y Rafael Castejón, decano de la Facultad de Ciencias Económicas de la Universidad Nacional a Distancia, realizaron un análisis sobre las «Fuentes documentales para el estudio del sector financiero en Andalucía», que fue expuesto por el primero de ellos. La amplitud del período abarcado 
-fines del siglo xviII al $\mathrm{xx}$ - y la extensión de la región estudiada justifican el hecho de que los autores hayan escogido la vía de la descripción minuciosa de los fondos por provincias, destacando aquellas sociedades bancarias cuyos archivos se conservan y conocen.

Fuera del programa, Agustín Guimerá, colaborador científico del Consejo Superior de Investigaciones Científicas, presentó una comunicación sobre «Las fuentes documentales para la historia de la banca en Canarias».

La tercera sesión de trabajo se celebró el día 27 por la mañana y su tema fue «Archivos de bancos de ámbito nacional». Tuvo por moderador a Pedro Tedde e intervinieron Pablo Martín Aceña, Gabriel Tortella, Elisa García Morales, Consuelo Varela Ortega y Teresa Tortella.

Pablo Martín Aceña, profesor titular de Historia Económica de la Universidad de Alcalá de Henares, expuso el contenido de su trabajo inédito sobre la sucursal del Banco de Bilbao en Londres, poniendo de relieve las diferencias existentes entre la estrategia financiera del citado banco español y la que habitualmente seguía la banca londinense. Describió las principales fuentes utilizadas que consultó, tanto en el Archivo del Banco de Bilbao como en el archivo de la propia sucursal.

Gabriel Tortella, catedrático de Historia Económica de la Universidad de Alcalá de Henares, habló de su reciente investigación encargada por el Banco de Crédito Industrial sobre la historia del mismo. Este ha sido un trabajo realizado en equipo, con un colaborador - Juan Carlos Jiménez- para la parte de investigación y redacción propiamente dicha y con un grupo de documentalistas para la labor de preparación de los fondos de archivo. Elisa García Morales, documentalista, completó la intervención de Gabriel Tortella con una exposición detallada sobre el Archivo del Banco de Crédito Industrial.

Consuelo Varela Ortega, profesora titular de Economía Agraria de la Escuela de Ingenieros Agrónomos de Madrid, expuso seguidamente su ponencia titulada «Estudio econométrico del mercado y los precios de la tierra: una experiencia en la utilización de los Archivos del Banco Hipotecario y del Banco de Crédito Agrícola». En ella se refirió al estado en que se encuentran estos dos archivos y las posibilidades que ofrecen para estudios sobre precios de la tierra.

Teresa Tortella, directora del Archivo del Banco de España, cerró el turno de ponencias de la sesión con el relato de las actividades desarrolladas por este archivo durante los cuatro años transcurridos desde el I Congreso. En su intervención, titulada «El Archivo del Banco de España: cuatro años después», informó acerca de las innovaciones y progresos llevados a cabo por este centro, refiriéndose con detenimiento a las secciones que lo integran $\mathrm{y}$, de un modo especial, a las que no habían sido descritas hasta ahora. 
La cuarta y última sesión de trabajo tuvo lugar el día 27 por la tarde. Se dedicó al tema «Archivos financieros de ámbito internacional» y actuó como moderador de la misma Gabriel Tortella. Intervinieron como ponentes los profesores Hans Pohl, Albert Broder y Rondo Cameron.

Hans Pohl, catedrático de Historia Económica de la Universidad de Bonn, de la República Federal de Alemania, habló sobre «Inversiones de capital en España hasta la Primera Guerra Mundial: datos y fuentes de archivos alemanes públicos y de empresas»; Albert Broder, catedrático de Historia Económica de la Universidad de Lille (Francia), se refirió a las «Fuentes para la historia económica española en archivos públicos y privados de Francia, Bélgica e Inglaterra», y Rondo Cameron, catedrático de Historia Económica de la Universidad de Emory (Atlanta, EE UU), completó las palabras de Albert Broder relatando algunas experiencias personales en sus investigaciones por archivos económicos europeos. Dieron, en conjunto, una visión de las fuentes sobre entradas de capital extranjero para determinados sectores de la economía española en el pasado reciente.

Pedro Tedde realizó a continuación un resumen del Congreso en el que se refirió a cada una de las ponencias e intervenciones, destacando los puntos más llamativos de ellas. Recapituló, asimismo, las principales conclusiones que se pueden deducir de este encuentro, subrayando, ante todo, algunas proposiciones de carácter general, que son las siguientes:

1. Petición unánime de que desde el Banco de España se exhorte a las entidades de crédito privadas para que rescaten y ordenen sus archivos históricos y los pongan a disposición de los investigadores con determinadas condiciones.

2. Que se establezca, a partir de la propia experiencia del Archivo del Banco de España, una normativa o guía para la selección de documentos dignos de conservarse en las empresas, así como para la organización y mantenimiento de los mismos.

3. Conveniencia de que se intercambie mayor información, de manera regulada, entre el Archivo del Banco de España y los de la Administración del Estado, a fin de coordinar la ordenación de determinados documentos de origen común o similar, repartidos entre estas instituciones.

4. ${ }^{a} \quad$ Posibilidad de que parta de los propios historiadores la iniciativa de orientar a las empresas actuales acerca de los criterios que deben seguir para seleccionar los documentos dignos de perpetuarse y necesidad de guardar convenientemente los archivos mecanizados.

5. Imperiosa necesidad de que cunda, en el seno de la Administración y de las empresas públicas, el cumplimiento de la Ley de 1985, del Patrimo- 
nio Histórico-Español, en aquellos artículos que se refieren a la conservación y ordenación de los documentos utilizados o emanados de las autoridades públicas que cesan en sus cargos.

Las Actas del I Congreso sobre Archivos Económicos de Entidades Privadas fueron publicadas por el Archivo del Banco de España en 1983. También en esta ocasión se van a editar las Actas de este II Congreso, que aparecerán probablemente a principios de 1987 . Las personas interesadas pueden solicitarlas al Servicio de Publicaciones del Banco de España. 